\title{
Neuropharmacologic Approaches to Restore the Brain's Microenvironment
}

\author{
Weizhe $\mathrm{Li}^{1}$ • Hsin-I Tong ${ }^{2} \cdot$ Santhi Gorantla ${ }^{1}$ - Larisa Y Poluektova ${ }^{1}$. \\ Howard E Gendelman ${ }^{1}$ - Yuanan $\mathrm{Lu}^{2}$
}

Received: 22 March 2016 / Accepted: 25 May 2016 /Published online: 28 June 2016

(C) Springer Science+Business Media New York 2016

\begin{abstract}
Maintaining the central nervous system microenvironment after injury, infection, inflammatory and degenerative diseases is contingent upon adequate control of glial homeostatic functions. Disease is caused by microbial, environmental and endogenous factors that compromise ongoing nervous system functions. The final result is neuronal injury, dropout and nerve connection loss, and these underlie the pathobiology of Alzheimer's and Parkinson's disease, amyotrophic lateral sclerosis, stroke, and bacterial, parasitic and viral infections. However, what promotes disease are homeostatic changes in the brain's microenvironment affected by innate glial immune pro-inflammatory and adaptive immune responses. These events disturb the brain's metabolic activities and communication abilities. How the process affects the brain's regulatory functions that can be harnessed for therapeutic gain is the subject at hand. Specific examples are provided that serve to modulate inflammation and improve disease outcomes specifically for HIV-associated neurocognitive disorders.
\end{abstract}

Keywords Astrocytes $\cdot$ Microglia $\cdot$ Neuroinflammation · Human immunodeficiency virus-associated neurocognitive disorders $\cdot$ Neurodegenerative disorders $\cdot$ Alzheimer's disease $\cdot$ Parkinson's disease

Howard E Gendelman

hegendel@unmc.edu

Yuanan Lu

yuanan@hawaii.edu

1 Department of Pharmacology and Experimental Neuroscience, University of Nebraska Medical Center, Omaha, NE 68198, USA

2 Department of Public Health Sciences, Environmental Health Laboratory, University of Hawaii at Manoa, Honolulu, HI 96822, USA

\section{Introduction}

For infectious, degenerative and inflammatory disorders of the central nervous system (CNS), microglial responses directly participate in disease. This occurs as a consequence of the cells' reaction to infectious, immune and degenerative processes (Perry et al. 2010; Streit et al. 1988). First, viral infections, for example, can lead to a parainfectious encephalomyelitis. This is commonly referred to as an acute disseminated encephalomyelitis (ADEM) where disease is driven pathobiologically by antigen-antibody attack. Autoimmunity occurs as a consequence of generalized neuroinflammation in the brain and spinal cord with profound myelin damage and white matter destruction. ADEM is commonly triggered after infection with a spectrum of viruses that include, but are not limited to influenza, measles, mumps, rubella, varicella zoster, Zika, Epstein Bar, cytomegalo-, entero- and herpes simplex viruses. ADEM can also be driven by vaccinations (Hemachudha et al. 1987; Ozawa et al. 2000). In both instances, brain injury is commonly self-limited, and permanent neurological sequel can occur but not commonly. Second, direct viral invasion of the CNS induces disease as a consequence of productive infection in neurons or accessory glial or endothelial cells. The process is active within the nervous system parenchyma. Such invasion is dependent on the pathogen, the immune status of the host and related genetics, and host immune responses. Such pathogenic events are commonly associated with significant morbidity and mortality. The former is seen with altered levels of consciousness and focal neurological symptoms commonly include seizures (Whitley 1990). A third cause of encephalitis is linked to inflammatory responses that occur as a consequence of viral infection of accessory cells and notably, monocytes, macrophages and microglia (He et al. 1997; Koenig et al. 1986). This directly involves neuronal destructive activities induced by viral and cellular neurotoxic proteins (Sørensen et al. 2008). A singular example of accessory cell 
infection-associated encephalitis is human immunodeficiency virus type one (HIV-1) infection. Apropos of HIV, a vigorous innate immune response occurs as a direct consequence of brain mononuclear phagocytes (MP; monocyte, perivascular macrophage and microglial) infection that drives pro-inflammatory and virotoxin-associated neurotoxic responses (Koenig et al. 1986; Persidsky and Gendelman 2003). This is perpetuated by autocrine and paracrine amplification of cytokine activities and by ongoing infection in the setting of a failure of immune responses or in patients where antiretroviral therapy has not been initiated (Schifitto et al. 2007). Neuronal loss in HIV-1 encephalitis is strongly associated with synaptic and dendritic damage (Masliah et al. 1996). Overt synaptic loss is in turn linked to severe cognitive impairment and brain viral load. Interestingly, levels of virus can vary dependent on brain region and underlies that it is the inducer of immune secretory factors, the perpetrators of disease (Kumar et al. 2007). Indeed, the development of dementia commonly but not always is associated with virus (Gray 1999). Interestingly, compensatory immune responses can restrict virus and attenuate disease; also, the advent of combination antiretroviral therapy has limited viral infection in the brain and facilitated host antiretroviral activities. Nonetheless, given the limited penetrance of antiretroviral drugs across blood-brain barrier (BBB) and comorbid conditions such as aging, drugs abuse and coinfection of hepatitis viruses in the HIV-infected population (Devlin et al. 2011), persistent neuroinflammation and viral reservoirs hiding in the brain remain to prevent the CNS function from restoring (Alexaki et al. 2008; Thompson et al. 2011).

For the degenerative Alzheimer's and Parkinson's diseases (AD and PD), the accumulation of misfolded, oxidized and aggregated proteins affect neuroinflammation and rapidly lead to neuronal dysfunction (Lashley et al. 2015; Polymeropoulos et al. 1997; Spillantini et al. 1997). Control of disease can also be associated with inflammation and immunity but how this occurs and can be harnessed for therapeutic gain is less clear (McGeer and McGeer and EG 2004; Salminen et al. 2009). In general terms, attenuation of inflammation either by drugs or by relevant adaptive immune responses can facilitate disease outcomes, but the corollary provides opposite endpoints. Altogether, with the importance of neuroinflammation as a cause for a range of disorders of varying etiology, a primary therapeutic goal is the control of brain's microenvironment regardless of the disease. Obviously, the removal of the root cause such as the infectious agent or the misfolded proteins remains primary. This can be realized by therapeutics and vaccination strategies (Benner et al. 2004; Buchbinder et al. 2008; Doody et al. 2014; Masliah et al. 2005; Morgan et al. 2000; Schneeberger et al. 2015). However, what must follow is the restoration of the brain's innate immune function in sustaining its metabolic function and homeostasis. This is what underlies restorative neuronal vitality (Benarroch 2005; Ransohoff and Brown 2012).
Here, we discuss the means to achieve the goals of controlling the brain's microenvironment. This will be highlighted through others and our own developments for therapeutics that are aimed towards restoring neural homeostasis. Underpinning this goal is a singular limitation that rests in drug penetrance across the BBB and as such access of therapeutic agents to damaged brain areas (Pardridge 2005). To this end, questions still exist on how BBB disruption occurs and can be reversed, how altering vascular channels can occur, and how chemical modification of drugs can be better developed to maximize therapeutic delivery (Baseri et al. 2012; Gabathuler 2010; Meairs and Alonso 2007; Wolak and Thorne 2013). Although each approach has its limitations and drawbacks, several chemical modification of drugs to increase lipophilicity to move through lipophilic BBB, disguising them with lipophilic molecules to make pro-drugs, attaching targeting molecules to get the drugs to pass through the BBB, and packaging them into nanoparticles or exosomes were investigated (Dash et al. 2011; El-Andaloussi et al. 2012; Hall et al. 2016; Pathan et al. 2009). Yet another means rests in the delivery system where we can harness MP as carriers of antiviral drug nanoparticles as well as disease fighting proteins and nucleic acids (Mallapragada et al. 2015). The delivery of such agents across the BBB remains a singular goal (Brynskikh et al. 2010; Dou et al. 2009; Nowacek and Gendelman 2009; Zhao et al. 2011). Overall, we discuss the feasibility of modulating the CNS microenvironment for therapeutic benefit. We also reviewed the recent advances in delivery of therapeutic agents to the brain with a particular emphasis on the means to restore the brain's microenvironment during disease using a spectrum of adjunctive therapies that include those that attenuate inflammation and deliver growth factors to the sites of injury (Biju et al. 2010; Eggert et al. 2010; Marker et al. 2013; Zhao et al. 2014).

\section{Attenuating Neuroinflammation}

Neurodegenerative conditions like AD, PD and HAND (HIVassociated neurocognitive disorders) are accompanied by over active inflammatory responses that herald onset and tempo of disease progression (Combs et al. 2000; Harezlak et al. 2011; McGeer and McGeer and EG 2004). The etiologies of each of these disorders reside in distinct agents that include aggregation of misfolded protein, immune attack and viral infection that lead to a commonly deleterious consequence that interrupts the homeostasis of the local microenvironment. Microglia, a sensor and scavenger for pathological events in the CNS, become rapidly activated in response to environmental, infectious and immunotoxin signals. These include in the case of neurodegenerative diseases with misfolded proteins. Microglia subsequently undergo a series of morphological, molecular and secretory changes that affect the brain's microenvironment (Aguzzi et al. 2013; Cameron and Landreth 2010; Kim and Joh 2006). Additionally, the 
interplay between microglia and astrocytes further polarizes the former towards a neurotoxic phenotype (Shih et al. 2006; Verderio and Matteoli 2001). Such changes in phenotype can affect the brain's microenvironment by producing a proinflammatory milieu that speeds neuronal injury and ongoing disease pathogenesis (Heneka et al. 2014). Although the CNS was historically considered to be immune-privileged, activation and transformation of resident and circulating immune cells in pathogenesis of neurodegenerative diseases indicate that brain hemostasis and immune system status are tightly coupled. Evidence also suggests that there are lymphoid controls operative in the brain (Wilson et al. 2010). Nonetheless, MP activation and subsequent induction of chemokines like CCL2, CXCL8 and pro-inflammatory mediators such as inducible nitric oxide synthase (iNOS), tumor necrosis factor- $\alpha$ (TNF- $\alpha$ ) and interleukin-6 (IL-6) collectively serve to speed the infiltration of circulating immune cells into the CNS (Czlonkowska et al. 1999; Rivest 2009) (Fig. 1a). The migration of effector memory $\mathrm{T}$ cells from the periphery generates leukocyte-microglial crosstalk that exacerbates neuroinflammation and restricts neuronal survival (Mallapragada et al. 2015). Another important player of immune origin contributing to the pro-inflammatory signals is mast cells, which are found in most tissues; they transverse compromised BBB to become CNS residents. Mast cell-glia communication also opens new perspectives for the development of therapies targeting neuroinflammation by differentially modulating activation of non-neuronal cells that normally control neuronal sensitization (Skaper et al. 2014). Immunopharmacologics are being developed as neuroprotective strategies for disease either to block the attraction of the circulating immune cells, $\mathrm{T}$ cells, macrophages and mast cells to the CNS by restoring the $\mathrm{BBB}$ or to induce anti-inflammatory responses in the CNS (Wilson et al. 2010). Disease-modifying treatments can control inflammation and restore neural function (Fig. 1b, c). Such adjunctive therapies take into account that modulating both innate and adaptive immune responses are necessary for success in ameliorating an inflammatory CNS microenvironment and as such restore neuronal function during disease (Popovich and Longbrake 2008).

The pragmatic question that is continuous over many years is why have so many anti-inflammatory therapies failed to improve disease outcomes for neurodegenerative disorders? (Nowacek et al. 2009). This is believed to be due to the overall pro-inflammatory microenvironment that remained uncontrolled or even deteriorated as diseases progress, which counteract the benefit from the blockage of over-activated excitatory synapses. In this regard, comprehensive management of neuroinflammation needs be taken at a more fundamental level. MP, microglia and macrophages, activated by HIV-1 facilitate neuroinflammation by upregulating pro-inflammatory cytokines and monocyte-chemotaxis signals, which in turn further recruit circulating MP to the diseased brain (Williams et al. 2012). For example, for HAND, attempts were made to use memantine, an uncompetitive antagonist of the N-methylD-aspartate (NMDA) receptor, as an adjunctive agent to antiretroviral therapies aimed at reducing excitatory neurotoxicity, failed to improve cognitive function (Schifitto et al. 2007). This was seen in a phase II double-blinded, randomized, placebo- controlled trial, despite positive preclinical investigations (Anderson et al. 2004).

Another example is with a mixed lineage kinase-3 (MLK3) inhibitor. MLK3, a widely expressed kinase in neurons (Maroney et al. 2001), astrocytes (Falsig et al. 2004), microglia (Hidding et al. 2002) and myeloid lineage cells (Handley et al. 2007), regulate the c-Jun N-terminal Kinase (JNK) MAPK and p38 signaling pathways, which is tightly related to monocyte chemotaxis via CCL2 and CXCL8 and to cytokine production by microglia and brain macrophages (Ahmed et al. 2009; Young and Arndt 2009; Zheng et al. 2008). It has been demonstrated that HIV Tat and gp120 protein can induce autophosphorylation at the activation loop of MLK3 in primary rat neurons and human monocytes, which can be abolished by CEP-1347, a large molecular inhibitor of MLK3 (Sui et al. 2006). Further in vivo studies also indicated the neuroprotective effect of CEP-1347 with apparent reduction in microgliosis, neuronal loss and preserved dendritic integrity in an HIV encephalitis (HIVE) model (Eggert et al. 2010). The neuroprotective attribute of CEP-1347 has also been shown in studies of other neurodegenerative disorders such as Huntington's disease (Apostol et al. 2008), AD (BozyczkoCoyne et al. 2001) and PD (Lotharius et al. 2005; Mathiasen et al. 2004; Saporito et al. 1999; Saporito et al. 2002). The promising results obtained in animal models of neurodegenerative disorders stretched to CEP-1347 clinical trials in PD patients. However, a phase II clinical trial concluded CEP1347 as ineffective treatment in early PD (Investigators 2007). Symptoms of PD start when about $70-80 \%$ of striatal dopamine and about half of nigral dopamine neurons have been lost (Bernheimer et al. 1973; McGeer et al. 1988). Neuronal loss occurs generally over the course of a preclinical period of several years (Dunnett and Bjorklund 1999). Therefore, the irreversible degeneration may have started before CEP-1347 could be given in this set of patients; moreover, single neuroprotective agent may not be sufficient to reverse the disease progression. Implementation of strategies for primary prevention will require the identification of markers that would permit early diagnosis prior to the onset of symptoms. Another concern resides in the limited distribution of CEP-1347 to the CNS due to its naturally polar structure and large molecular weight (Kaneko et al. 1997). Indeed, a new MLK-3 inhibitor, URMC-099, with a favorable chemical profile and CNS penetrant property was designed. This compound demonstrated a significant amount of the neuroprotection imparted by inhibiting MLK3 in immune cells and microglia (Marker et al. 2013). 

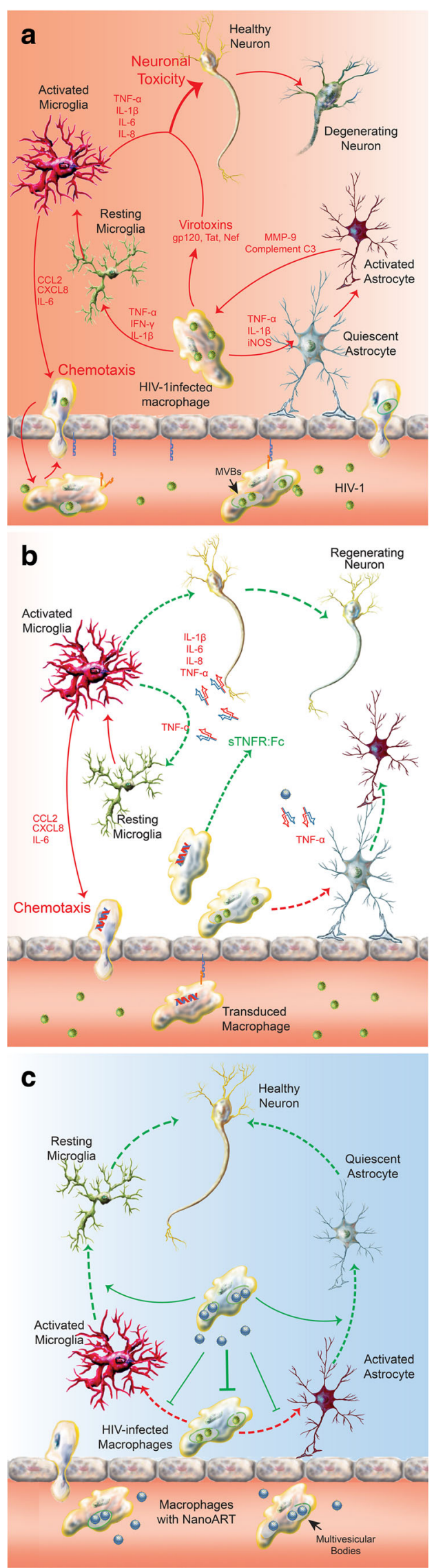

Fig. 1 Combination of adjunctive and antiretroviral treatments for HAND. A vigorous innate immune response occurs as a direct consequence of brain mononuclear phagocytes (perivascular macrophages, microglia) infection that drives a pro-inflammatory and virotoxin-associated neurotoxic response. This is perpetuated by an autonomous amplification of cytokine activities like TNF- $\alpha$ and by a persistent chemotaxis of HIV-1 infected macrophages from periphery in the setting of a failure of immune responses or an absence of antiretroviral therapy. Meanwhile HIV-1 infected macrophages interrupt astroglial function and integrity by pro-inflammatory mediators, compromising the metabolic function and homeostasis of the CNS. Neuronal loss in HIV-1 encephalitis is associated with synaptic and dendritic damage. Overt synaptic loss is in turn linked to cognitive impairment induced by the brain's viral load (a). The chemokine gradient in diseased brain drives transduced macrophage constitutively expressing sTNFR:Fc to the inflammatory site. Local release of sTNFR:Fc reverses the pro-inflammatory microenvironment and blocks a cycle of autocrine and paracrine cytokines produced by sequestering the major pro-inflammatory mediator TNF- $\alpha$ (b). Delivery of nanoART using macrophage as carriage serves to control HIV-1 replication in the brain by bringing antiretroviral drugs to local sites of viral growth. The long-acting nanoformulated-antiretroviral medicines are in the same subcellular viral compartment within multivesicular bodies (MVBs). This serves to reduce viral loads and numbers of peripheral HIV-1 infected CD4+ T-cells. Drug levels are sustained in reservoirs of viral infection (c). Upon the suppression of persistent inflammation and clearance of viral brain reservoirs, the function and homeostasis of microglia, astrocyte and most neurons are maintained

The effectiveness of CEP1347 and URMC-099 in the control of neuroinflammation and protecting neurons from injury is significant with the latter showing dramatic effects in the treatment of experimental HAND models (Eggert et al. 2010; Marker et al. 2013). The known etiology and definite onset point of HAND possibly render this an advantage. Nevertheless, the power of these in vivo studies was partially limited until very recently when URMC-099 was shown to have both neuroprotective and unique antiretroviral effects (Zhang et al. 2016). Indeed, during studies to extend the half-life of crystalline nanoformulated antiretroviral therapy (nanoART), URMC-099, which was originally developed as an adjunctive neuroprotective agent, was demonstrated to broaden antiviral responses and improve ART biodistribution. Co-administration of long-acting ritonavir-boosted atazanavir (nanoATV/r) nanoformulations with URMC-099 not only reduced viral load and the numbers of HIV-1 infected CD4+ Tcells in lymphoid tissues but sustained drug levels in reservoirs of viral infection. These results were realized more than either drug alone when administered in infected humanized $\mathrm{NOD} / \mathrm{SCID} / \mathrm{IL} 2 \mathrm{R} \gamma \mathrm{c}-/-$ mice and paralleled the formation of drug depots within the reticuloendothelial system. The autophagosome appears to be that depot and to lead to the sequestration of nanoATV/r in Rab-associated recycling and late endosomes (Zhang et al. 2016). These results provide an example for how unique immune modulatory reactions can also improve antiretroviral responses. As such the drug 
combinations are being developed beyond protease inhibitors to involve other drug classes that include integrase inhibitors to facilitate cell-based viral clearance.

\section{Cell-Based Gene and Immune Modulatory Therapies}

Well known is the fact that MP can cause BBB breakdown following brain inflammation, migrating toward the inflammation site via processes known as diapedesis and chemotaxis (Woodward and Troedsson 2015). Relying on the natural inflammatory responses, macrophages have been widely applied for the active delivery of therapeutic agents, including nucleic acids, peptides, drugs and enzymes, to the brain. Indeed, delivery of nanoformulated antiretroviral drugs (nanoART) using macrophages as carriers has significantly increased drug concentration and reduced HIV-1 replication in the diseased compartments of HIVE rodents (Dou et al. 2009). Limited effect on the ongoing neuroinflammation in this study, however, reinforced the importance of combining immune-modulatory therapy to restore the brain's innate immune and metabolic homeostasis.

Gene therapy is the therapeutic approach of precise manipulation of the host genome, ultimately the function of targeted cells. Recent studies and clinical trials have employed a variety of vehicles to deliver protective genes to the CNS (Dey et al. 2010; Duebgen et al. 2014; Munoz et al. 2013; Zappia et al. 2005) (NCT01446614, NCT01883661). With the known effectiveness of soluble TNF-receptor (sTNFR) in the treatment of peripheral autoimmune and inflammatory conditions including rheumatoid arthritis (Moreland et al. 1999), we reasoned that a parallel approach could be applied for HIV infections of the nervous system where a pro-inflammatory environment seen within the brain incites neuronal-drop out and related cognitive dysfunction. We showed that the bicistronic plasmid construct, sTNFR-Fc-eGFP, expressing the sTNFR-Fc fusion protein and the green fluorescent protein (GFP) was suitable for stable expression of sTNFR-Fc in human macrophages and neuronal cells as a potential therapy for neuroAIDS (Cao et al. 2011). To this end, we transduced bone marrow-derived macrophages (BMDM) with the defective lentiviral vector encoding sTNFR:Fc-eGFP (MOI =15). In BMDM sTNFR-GFP (below this used as shorter abbreviation of transcript) was expressed with efficiency greater than $45 \%$ of transduced cells (Fig. 2a). The transduction efficiency of the injected BMDM was confirmed by flow cytometry (ten days post transduction), which demonstrated on average more than $45 \%$ of BMDM were GFP+ (Fig. 2b). Two hours prior to adoptive transfer of the recombinant sTNFR-GFP transduced BMDM, the immune deficient NOD.Cg-Prkdc ${ }^{\text {scid }}$ $I l 2 \mathrm{rg}^{t m 1 \mathrm{Wjl} /} / \mathrm{SzJ}$ (NSG) mice were injected intracranially (i.c.) in the caudate putamen with $2 \times 10^{5} \mathrm{HIV}-1_{\mathrm{ADA}}$ infected monocyte-derived macrophages (MDM) as described previously (Dou et al. 2009). This procedure induced encephalitis referred to as HIVE, which paralleled what is operative, in infected patients (Fig. 2c).

Adoptive transfer of sTNFR transduced BMDM showed no change in numbers of HIV-1p24 positive infected cells or the intensity of astrocyte responses, but a modest reduction in microglial staining was evident (Fig. 3a). In this survey histopathological analyses of brain sections were acquired at sites of HIV-infection-mediated injury. Here, Iba-1 staining showed that adoptive transfer of sTNFR-GFP transduced BMDM attenuated microgliosis in brain areas surrounding the injection sites of HIV-1 infected MDM, when compared to adoptive transfer of control BMDM. In Fig. 3b, neurofilament staining was evaluated in and around the injection site and quantitated by Nuance multispectral imaging system. Transduced HIV-1 infected mice have significantly higher NF content staining than control groups; GFAP intensities were not significantly different amongst the groups (Fig. 3b, lower panel). Parallel analyses of synaptic markers that include microtubule associated protein 2 (MAP-2) and synaptophysin (SYN) failed to demonstrate changes as a result of therapy and when quantitated by similar multispectral imaging markers (data not shown).

We next evaluated by real-time polymerase chain reaction (RT-PCR) the gene expression levels of sTNFR-Fc in the brain samples HIVE animals injected with transduced BMDM; pro-inflammatory cytokine and neurotrophic factor (GDNF and BDNF) expression levels were measured in brains of animals that received STNFR-Fc or control vector containing BMDM. Notably, expression of sTNFR-Fc was detected in the brains of animals that received transduced BMDM (Fig. 3c). No statistically significant differences in NOS2, IL-10, IL-1b, TNF- $\alpha$, BDNF and GDNF were noted between animals treated with transduced (TD) and nontransduced (NTD) BMDM (Fig. 3e). However, in these experiments measurement of pro- and anti- inflammatory and neurotrophic factors were also compared in brain tissues of HIVE controls (HIV-Ctl) with animals adoptively transferred with TD BMDM. Here, decreased expression of NOS2, IL-10, IL-1b and TNF- $\alpha$ shown in the TD animals compared to HIV-Ctl group (Fig. 3d). Adoptive transfer of macrophages in an HIVE experimental model might have by itself negative effects associated with additional influx of macrophages (with control vectors or non-transduced). Delivery of sTNFR by transduced BMDM at the initiation of inflammation showed positive anti-inflammatory effect. The reduction in microgliosis by sTNFR-GFP transduced BMDM and the induction of anti-inflammatory milieu might be contributing to the neuroprotective response with increased $\mathrm{NF}$ immunostaining around the injected area (Fig. 3b). 


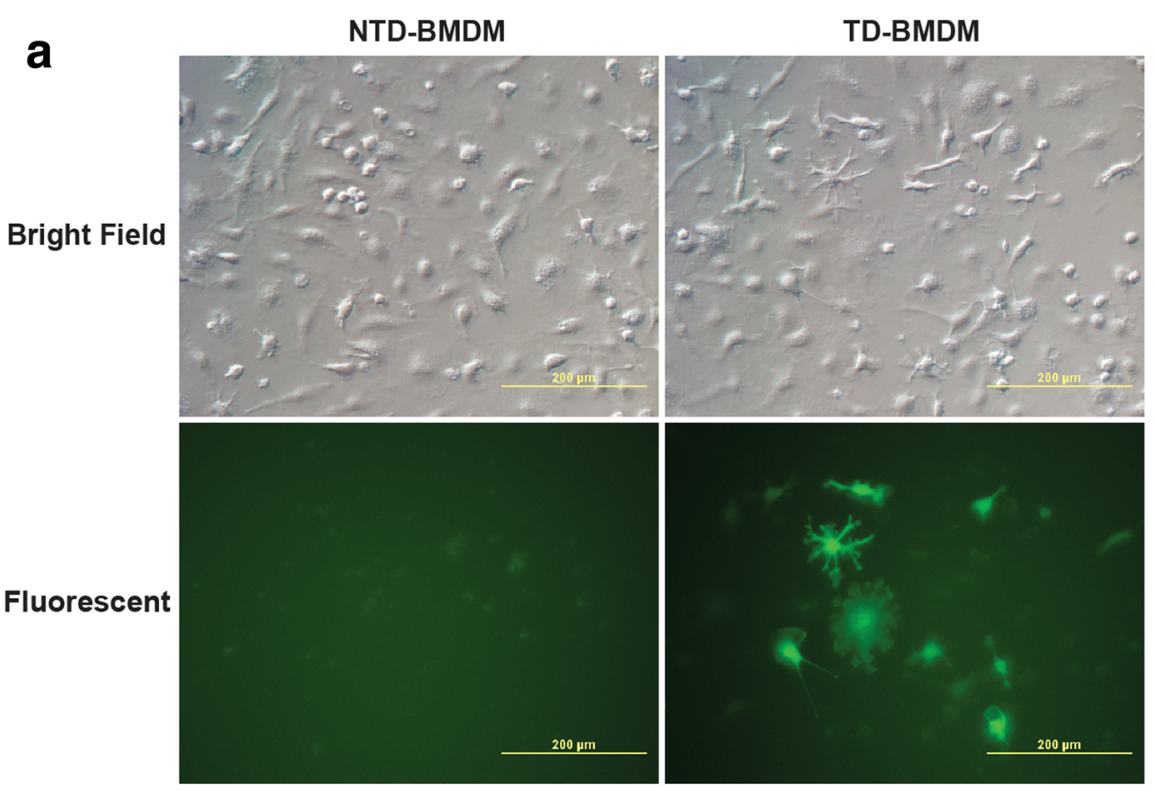

\section{b}

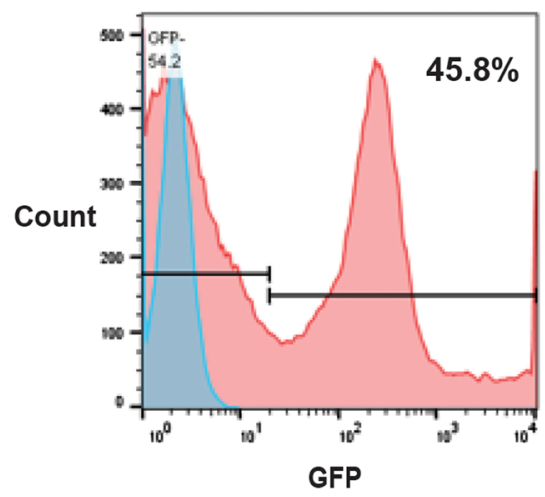

C

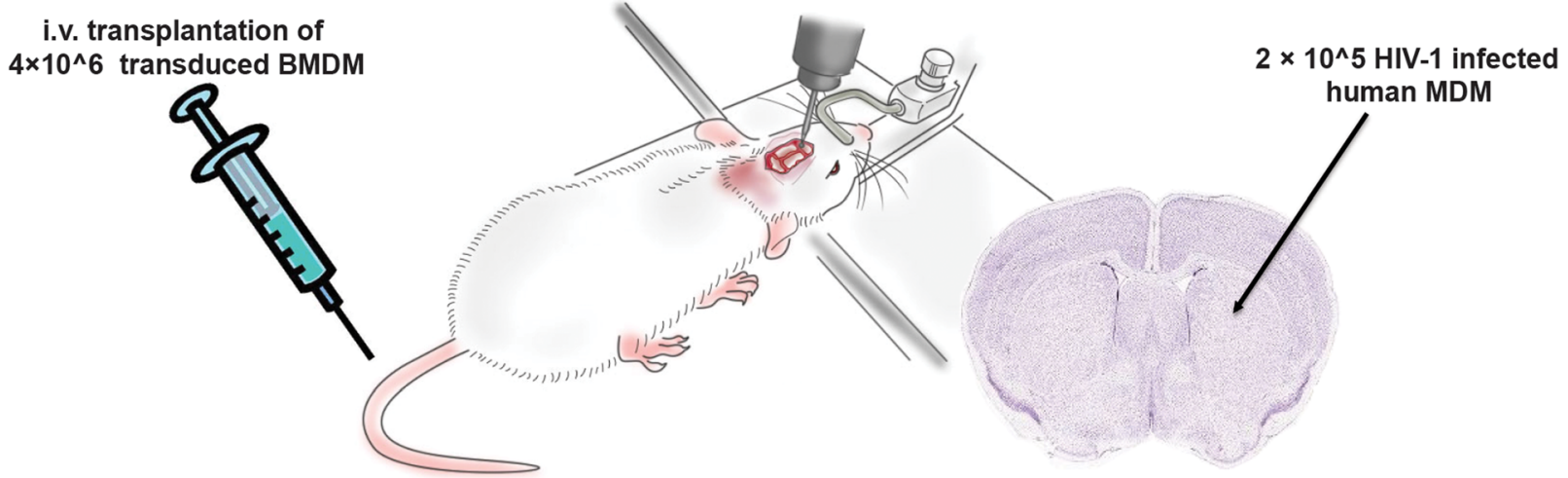

Fig. 2 Adoptive transplantation of sTNFR-GFP transduced BMDM into HIVE mice. BMDM transduced with the sTNFR-GFP at day 7 in vitro were shown positive for enhanced GFP expression and started to exhibit macrophage-like branching appearance. One of three representative experiments is shown (a). Transduction efficiency measured by flow cytometry ten days post transduction demonstrated more than $45 \%$ transduced $\mathrm{BMDM}$ were GFP+, one of three representative experiments is shown (b). Four-week-old female NSG mice were stereotactically injected intracranially into the caudate putamen with $\mathrm{HIV}-1_{\mathrm{ADA}^{-}}$

A similar approach has been applied in the PD study to deliver neurotropic and protective factors to attenuate neuroinflammatory processes linked to neuronal death (Zhao et al. 2014). Glial cell line-derived neurotrophic factor (GDNF) is the most potent survival factor for the nigrostriatal dopaminergic neurons that degenerate in PD (Bjorklund et al. 1997; Gash et al. 1996; Kordower et al. 2000; Pascual et al. 2008; Ramaswamy et al. 2009). Current GDNF-based therapies have been seriously hindered by the difficulty in delivering these therapies to the brain. Moreover, the progressive nature of PD requires continuous and sustained delivery of infected MDM $\left(2 \times 10^{5}\right.$ cells in $\left.5 \mu \mathrm{l}\right)$ after $1 \mathrm{~d}$ of viral infection and referred to as HIVE mice (c). These mice were subsequently transplanted with $4 \times 10^{6}$ transduced (TD, $n=12$ ) or non-transduced (NTD, $n=10)$ BMDMs in $200 \mu \mathrm{l}$ PBS through tail vein. HIVE controls (HIV-Ctl) were injected with same volume of PBS $(n=8)$; SHAM controls were only intracranially injected with $5 \mu \mathrm{PBS}(n=6)$. The mouse sketch was provided by Encapsula NanoSciences with copyright permission

GDNF over months or years in order to maintain dopamine neuron survival and function. Conjugation of GDNF with other molecules enabling BBB penetration may partially overcome these difficulties and increase the feasibility of this approach for long-term sustained delivery of GDNF remains uncertain. Advancement in the development of cell-based gene therapy that can be applied systemically and achieve sustained release of moderate amounts of GDNF opened a new window (Fig. 4). Adoptive transfer of genetically modified macrophage expressing GDNF dramatically ameliorated neuroinflammation and reduced degeneration of the tyrosine 

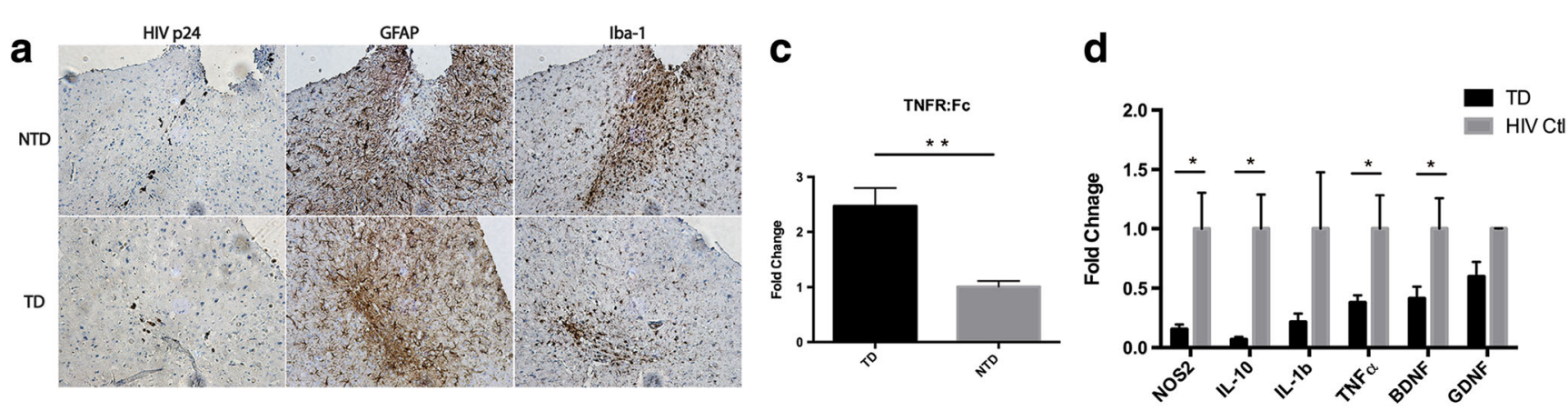
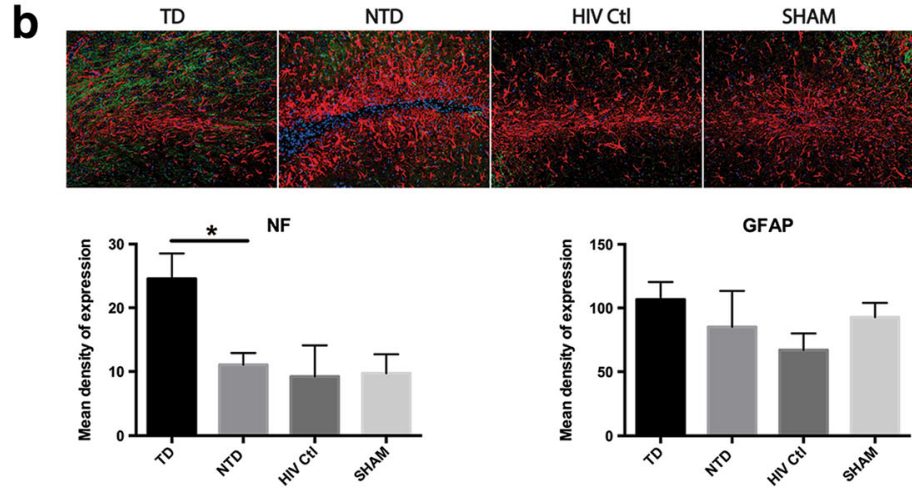

Fig. 3 Delivery of sTNFR by transduced BMDM demonstrated an antiinflammatory effect in an HIVE model. Immunohistochemical staining of HIV p24 protein identified HIV-1 infected human MDM at injection site. Evaluation of astrogliosis and microgliosis by GFAP and Iba-1 staining revealed adoptive transplantation of sTNFr:Fc-GFP transduced BMDM attenuated the microgliosis surrounding injection site compared to transplantation of NTD cells. But no apparent reduction of astrogliosis was observed (a). GFAP/Neurofilament staining in the injection site were analyzed by Nuance multispectral imaging system (20X objective). The representative images are shown. Transduced group have significantly higher content of NF (green) staining in comparison with other groups. However, the difference in the intensity of GFAP was not statistically significant among groups (b). The expression of genes encoding TNFR:Fc, NOS2, IL1- $\beta$, TNF- $\alpha$, NOS2, BDNF, and GDNF in brain subregions containing injection site were measured by real-time RT-PCR using SYBR green system and specific primers. $n=5$ in TD group, $n=6$ in NTD group, $n=3$ in HIV Ctl group. The expression of TNFR:Fc is more than two-fold higher in TD than in NTD brain (c). The expression of

hydroxylase positive $\left(\mathrm{TH}^{+}\right)$neurons of the substantia nigra and $\mathrm{TH}^{+}$terminals in the striatum of the 1-methyl-4-phenyl1,2,3,6-tetrahydro-pyridine (MPTP) and the 6hydroxydopamine (6-OHDA) PD mice models (Biju et al. 2010; Zhao et al. 2014). Genetically modified macrophages acting as carriages for GDNF were recruited into the substantia nigra of MPTP-treated mice, both the number of dopaminergic neurons and the level of dopamine were rescued in the striatum of MPTP-treated GDNF mice (Biju et al. 2010). In another study, exosomes released from GDNFtransfected macrophages resulted in the drastic increase of neuronal maturation with a pronounced outgrowth of axons and dendrites in PC12 neurons in vitro (Zhao et al. 2014). This attributes to the potential mechanism of efficient interactions between exosomal carriers and plasma membranes of inflammatory cytokines including NOS2, IL-10, IL- $1 \beta$, TNF- $\alpha$ and neurotrophic factors like BDNF and GDNF, is lower in TD than they are in HIV-Ctl brain (d). No significant differences in expression in TD group when compared to NTD group were found (e). For multispectral image quantifications, images were converted to 12 bit grayscale and quantified using ImagePro plus software. There are 3 animals in each group, and 4 sections of selected regions from each animal were analyzed and quantified. Each bar represented as mean \pm SEM, $P$ values were obtained using one way ANOVA test, ${ }^{*} p<0.05$. Antibodies used include mouse anti-Neurofilament (1:200, Clone 2F11, Dako, CA), polyclonal rabbit anti-GFAP (1:1000, Dako, CA), and rabbit anti- Ibal (1:500, Wako, VA). For real-time RT-PCR, Ct values of each target gene were normalized to values for GAPDH in each sample. To calculate relative amounts mRNA, the average $\mathrm{Ct}$ values were subtracted from GAPDH values for each target gene to provide changes in $\mathrm{Ct}$ value. Fold Change in expression was calculated as $\log _{2}$ relative units. Multiple $t$ tests using Holm-Sidak method were applied for statistical analysis. Each bar represent mean \pm SEM with $* p<0.05,{ }^{*} p<0.01$ dopaminergic neurons that facilitate GDNF binding to their receptors, resulting in the profound therapeutic effect. Nevertheless, due to the limited understanding, the mechanism responsible for the therapeutic effects of cell-based GDNF delivery remains inconclusive. Recently the hypothesis that growth factors could effectively protect from endoplasmic reticulum stress, augment autophagy and brake or significantly reduce speed of misfolded proteins accumulation in PD, HD, ALS and AD was reviewed (Cai et al. 2016; Garcia-Huerta et al. 2016). A comprehensive examination and detailed understanding of the mechanisms would be of great value in designing strategies for cell-mediated drug delivery.

As inflammation has been implicated as a critical mechanism in $\mathrm{AD}$ pathogenesis, suppression of microglial activation and subsequent neuroinflammation was investigated 


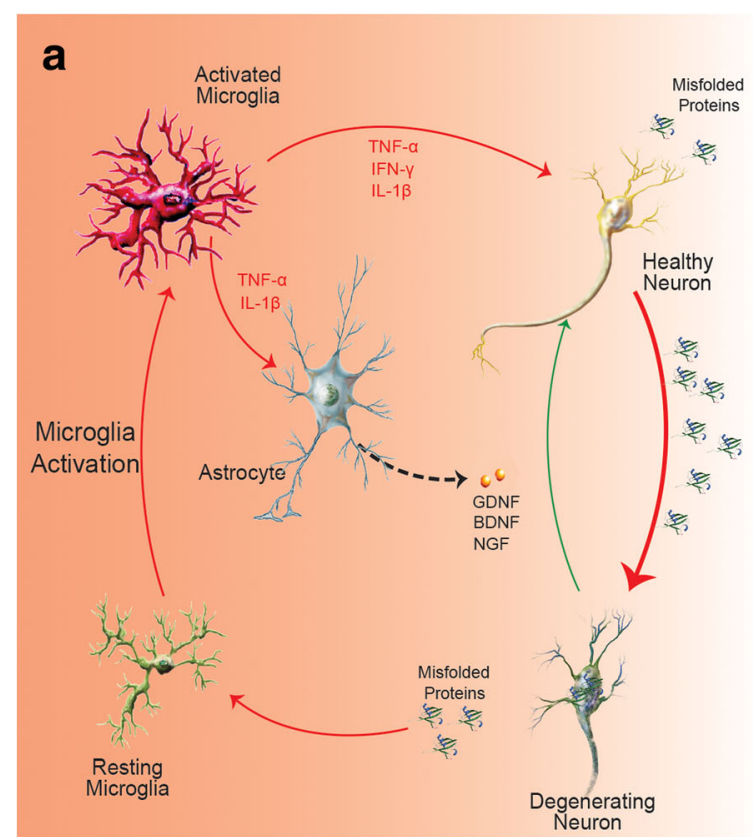

Fig. 4 Common mechanism of neurodegenerative pathology mediated by microglia and astrocyte activation, and reversal of neuronal dropout by introducing neurotrophic factors by transduced macrophages. Activated microglia undergo a series of morphological, molecular and secretory changes in the presence of abnormal proteins generated by neurons ( $\mathrm{a}-$ synuclein, amyloid etc.) that lead to a pro-inflammatory microenvironment in the CNS, and, as results progressively increasing build-up of misfolded proteins. These include the induction proinflammatory mediators such as IFN- $\gamma, \mathrm{NF}-\alpha$ and IL- $1 \beta$ speeding the neuronal dysfunction and degeneration. The interplay between microglia and astrocytes further impairs the astrocytes capability of maintaining

employing several anti-inflammatory drugs in trial, including natural nonsteroidal anti-inflammatory drugs (NSAIDs), polyphenols and new drugs synthesized based on multi-target directed ligand (MTDL) design (Shi et al. 2013). TNF-alpha inhibitor administered perispinally in patients with mild-tosevere AD showed promise as potential treatment approach (Tobinick et al. 2006). Adjunctive therapies targeting multiple factors in $\mathrm{AD}$ processes might be the best potential treatment strategy as in any other neurodegenerative disease.

\section{Conclusion}

In summary, this review and the preliminary data provided underscores the current approaches of modulating the altered microenvironment under various pathological conditions. With a primary goal of eradicating root causes of diseases, development of adjunctive therapies will not only enhance the efficacy of primary therapy but will contribute pivotally in restoring homeostasis and function of the CNS. For instance, transduced BMDM produced high levels of sTNFR in vitro. Adoptive

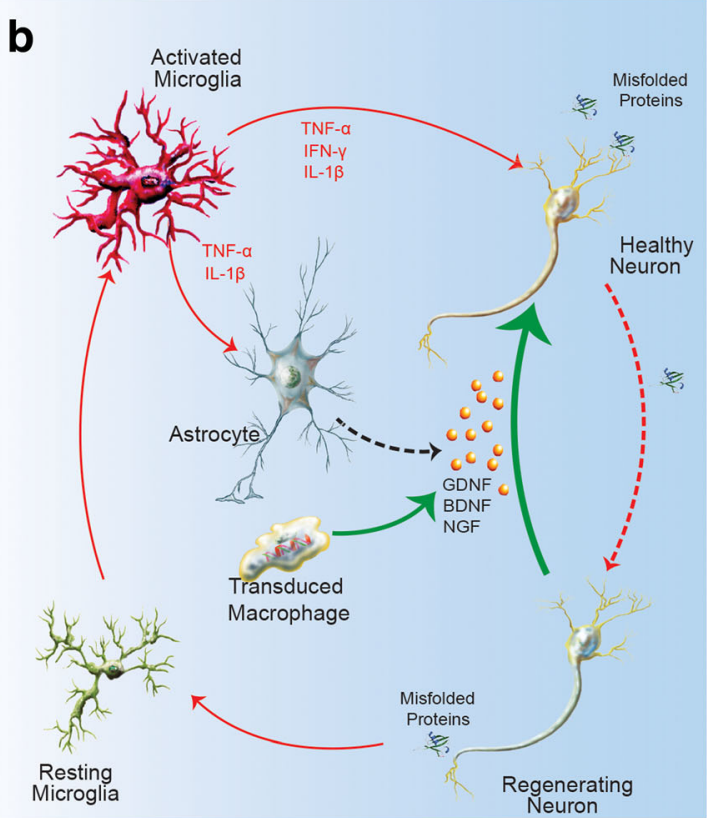

homeostasis and nourishing neurons with neurotrophic factors like GDNF, BDNF, and NGF (a). Adoptive transfer of genetically modified macrophage expressing neurotrophic factors dramatically ameliorated neuroinflammation and reduced degeneration of neurons in rodent models of $\mathrm{AD}$ and PD. Uptake of exosomes containing neurotrophic factors released from transduced macrophages resulted in a reduction of misfolded protein production via autophagy and should significantly delay their accumulation (b). The efficient interactions between exosomal carriers and plasma membranes of neurons that facilitate growth factor binding to their receptors may contribute to therapeutic end points

transfer of the transduced BMDM to a mouse model of HIVE demonstrated a trend for anti-inflammatory effects at disease site. Stable expression of immune modulatory peptides like sTNFR could readily work in conjunction with nanoART in dampening local inflammation. This suggested the future direction of using cell-based delivery of nanoART and neuroprotective peptide/proteins in achieving pathological and functional recovery simultaneously in HAND patients (Fig. 1b and c).

Parallel systems were devised for treatment of age-linked neurodegenerative disorders. This includes $\mathrm{AD}$ and $\mathrm{PD}$. The developed delivery systems are honed to overcome the BBB and deliver drugs to brain lesions. To rescue and recover the degenerating neurons, cell-based gene and peptide delivery could ensure a sustained supply of neurotrophic factors in the local microenvironment.

Acknowledgments This work was supported, in part, by the University of Nebraska Foundation, which includes but is not limited to individual donations from Carol Swarts, M.D., and Frances and Louie Blumkin, and the National Institutes of Health grants P01 DA028555, R01 NS36126, P01 NS31492, 2R01 NS034239, P01 MH64570, P01 NS43985, P30 MH062261 and R01 AG043540 (HEG); R24 OD 018546-01 (LP); and R01 MH079717 (YL). We thank the UNMC - Flow Cytometry Core 
facility for its help in data acquisition and analysis. We appreciate the Encapsula NanoSciences for providing the mouse artwork.

\section{Compliance with Ethical Standards}

Conflict of Interest The authors declare they have no conflicts of interest.

\section{References}

Aguzzi A, Barres BA, Bennett ML (2013) Microglia: scapegoat, saboteur, or something else? Science 339:156-161

Ahmed RAM, Murao K, Imachi H, Yoshida K, Dobashi H, Hosomi N, Ishida T (2009) c-Jun N-terminal kinases inhibitor suppresses the TNF- $\alpha$ induced MCP-1 expression in human umbilical vein endothelial cells. Endocrine 35:184-188

Alexaki A, Liu Y, Wigdahl B (2008) Cellular reservoirs of HIV-1 and their role in viral persistence. Curr HIV Res 6:388

Anderson ER, Gendelman HE, Xiong H (2004) Memantine protects hippocampal neuronal function in murine human immunodeficiency virus type 1 encephalitis the. J Neurosci 24:7194-7198

Apostol BL et al. (2008) CEP-1347 reduces mutant huntingtin-associated neurotoxicity and restores BDNF levels in R6/2 mice. Mol Cell Neurosci 39:8-20

Baseri B et al. (2012) Activation of signaling pathways following localized delivery of systemically administered neurotrophic factors across the blood-brain barrier using focused ultrasound and microbubbles. Phys Med Biol 57:N65

Benarroch EE (2005) Neuron-astrocyte interactions: partnership for normal function and disease in the central nervous system. In: Mayo Clinic Proceedings. Elsevier, 10:1326-1338

Benner EJ et al. (2004) Therapeutic immunization protects dopaminergic neurons in a mouse model of Parkinson's disease. Proc Natl Acad Sci U S A 101:9435-9440

Bernheimer H, Birkmayer W, Hornykiewicz O, Jellinger K, Seitelberger F (1973) Brain dopamine and the syndromes of Parkinson and Huntington. Clinical, morphological and neurochemical correlations. Journal of the neurological sciences 20:415-455

Biju K et al. (2010) Macrophage-mediated GDNF delivery protects against dopaminergic neurodegeneration: a therapeutic strategy for Parkinson's disease. Mol Ther: the J American Soc Gen Ther 18: 1536-1544. doi:10.1038/mt.2010.107

Bjorklund A, Rosenblad C, Winkler C, Kirik D (1997) Studies on neuroprotective and regenerative effects of GDNF in a partial lesion model of Parkinson's disease. Neurobiol Dis 4:186-200. doi:10. 1006/nbdi.1997.0151

Bozyczko-Coyne D, O'Kane TM, Wu ZL, Dobrzanski P, Murthy S, Vaught JL, Scott RW (2001) CEP-1347/KT-7515, an inhibitor of SAPK/JNK pathway activation, promotes survival and blocks multiple events associated with $\mathrm{A} \beta$-induced cortical neuron apoptosis. J Neurochem 77:849-863

Brynskikh AM et al. (2010) Macrophage delivery of therapeutic nanozymes in a murine model of Parkinson's disease. Nanomed: Nanotech biol Med 5:379-396

Buchbinder SP et al. (2008) Efficacy assessment of a cell-mediated immunity HIV-1 vaccine (the Step Study): a double-blind, randomised, placebo-controlled, test-of-concept trial. Lancet 372:1881-1893

Cai Y, Arikkath J, Yang L, Guo ML, Periyasamy P, Buch S (2016) Interplay of endoplasmic reticulum stress and autophagy in neurodegenerative disorders. Autophagy 12:225-244. doi:10.1080/ 15548627.2015.1121360
Cameron B, Landreth GE (2010) Inflammation, microglia, and Alzheimer's disease. Neurobiol Dis 37:503-509

Cao S, Wu C, Yang Y, Sniderhan LF, Maggirwar SB, Dewhurst S, Lu Y (2011) Lentiviral vector-mediated stable expression of sTNFR-Fc in human macrophage and neuronal cells as a potential therapy for neuroAIDS. J Neuroinflammation 8:48

Combs CK, Johnson DE, Karlo JC, Cannady SB, Landreth GE (2000) Inflammatory mechanisms in Alzheimer's disease: inhibition of $\beta$ amyloid-stimulated proinflammatory responses and neurotoxicity by PPAR $\gamma$ agonists. J Neurosci 20:558-567

Czlonkowska A, Czlonkowski A, Kohutnicka M, Kurkowska-Jastrzebska I, Wronska a (1999) MHC class II positive microglia and lymphocytic infiltration are present in the substantia nigra and striatum in mouse model of Parkinson's disease. Acta Neurobiol Exp 59:1-8

Dash PK et al. (2011) Loss of neuronal integrity during progressive HIV1 infection of humanized mice. J Neurosci 31:3148-3157

Devlin KN et al. (2011) Neurocognitive effects of HIV, hepatitis C, and substance use history. J Int Neuropsychol Soc 18:68

Dey ND et al. (2010) Genetically engineered mesenchymal stem cells reduce behavioral deficits in the YAC 128 mouse model of Huntington's disease. Behav Brain Res 214:193-200. doi:10.1016/ j.bbr.2010.05.023

Doody RS et al. (2014) Phase 3 trials of solanezumab for mild-tomoderate Alzheimer's disease. N Engl J Med 370:311-321

Dou $\mathrm{H}$ et al. (2009) Macrophage delivery of nanoformulated antiretroviral drug to the brain in a murine model of neuroAIDS. J Immunol (Baltimore, Md: 1950) 183:661-669. doi:10.4049/jimmunol.0900274

Duebgen M, Martinez-Quintanilla J, Tamura K, Hingtgen S, Redjal N, Wakimoto H, Shah K (2014) Stem cells loaded with multimechanistic oncolytic herpes simplex virus variants for brain tumor therapy. J Natl Cancer Inst 106:dju090. doi:10. 1093/jnci/dju090

Dunnett SB, Bjorklund a (1999) Prospects for new restorative and neuroprotective treatments in Parkinson's disease. Nature 399: A32-A39

Eggert D et al. (2010) Neuroprotective activities of CEP-1347 in models of neuroAIDS. J Immunol (Baltimore, Md: 1950) 184:746-756. doi:10.4049/jimmunol.0902962

El-Andaloussi S et al. (2012) Exosome-mediated delivery of siRNA in vitro and in vivo. Nat Protoc 7:2112-2126. doi:10.1038/nprot. 2012.131

Falsig J, Pörzgen P, Lotharius J, Leist M (2004) Specific modulation of astrocyte inflammation by inhibition of mixed lineage kinases with CEP-1347. J Immunol 173:2762-2770

Gabathuler R (2010) Approaches to transport therapeutic drugs across the blood-brain barrier to treat brain diseases. Neurobiol Dis 37:48-57

Garcia-Huerta P, Troncoso-Escudero P, Jerez C, Hetz C, Vidal RL (2016) The intersection between growth factors, autophagy and ER stress: a new target to treat neurodegenerative diseases? Brain Res. doi:10. 1016/j.brainres.2016.02.052

Gash DM et al. (1996) Functional recovery in parkinsonian monkeys treated with GDNF. Nature 380:252-255. doi:10.1038/380252a0

Gray F (1999) Neuronal apoptosis does not correlate with dementia in HIV infection but is related to microglial activation and axonal damage. Neuropathol Appl Neurobiol 25:123-133

Hall J, Prabhakar S, Balaj L, Lai CP, Cerione RA, Breakefield XO (2016) Delivery of therapeutic proteins via extracellular vesicles: review and potential treatments for Parkinson's disease. Glioma, and Schwannoma Cell Mol Neurol. doi:10.1007/s10571-015-0309-0

Handley ME, Rasaiyaah J, Barnett J, Thakker M, Pollara G, Katz DR, Chain BM (2007) Expression and function of mixed lineage kinases in dendritic cells. Int Immunol 19:923-933

Harezlak J et al. (2011) Persistence of hiv- associated cognitive impairment, inflammation and neuronal injury in era of highly active antiretroviral treatment. AIDS (London, England) 25:625 
He J et al. (1997) CCR3 and CCR5 are co-receptors for HIV-1 infection of microglia. Nature 385(6617):645-649

Hemachudha T, Griffin DE, Giffels JJ, Johnson RT, Moser AB, Phanuphak P (1987) Myelin basic protein as an encephalitogen in encephalomyelitis and polyneuritis following rabies vaccination. $\mathrm{N}$ Engl J Med 316:369-374. doi:10.1056/nejm198702123160703

Heneka MT, Kummer MP, Latz E (2014) Innate immune activation in neurodegenerative disease. Nat Rev Immunol 14:463-477

Hidding U et al. (2002) The c-Jun N-terminal kinases in cerebral microglia: immunological functions in the brain. Biochem Pharmacol 64: 781-788

Investigators PSGP (2007) Mixed lineage kinase inhibitor CEP-1347 fails to delay disability in early Parkinson disease. Neurology 69: $1480-1490$

Kaneko M et al. (1997) Neurotrophic 3, 9-bis [(alkylthio) methyl]-and-bis (alkoxymethyl)-K-252a derivatives. J Med Chem 40:1863-1869

Kim YS, Joh TH (2006) Microglia, major player in the brain inflammation: their roles in the pathogenesis of Parkinson's disease. Exp Mol Med 38:333-347

Koenig S et al. (1986) Detection of AIDS virus in macrophages in brain tissue from AIDS patients with encephalopathy. Science 233:10891093

Kordower JH et al. (2000) Neurodegeneration prevented by lentiviral vector delivery of GDNF in primate models of Parkinson's disease. Science 290:767-773

Kumar AM, Borodowsky I, Fernandez B, Gonzalez L, Kumar M (2007) Human immunodeficiency virus type 1 RNA levels in different regions of human brain: quantification using real-time reverse transcriptase-polymerase chain reaction. J Neurovirol 13:210-224. doi:10.1080/13550280701327038

Lashley T, Rohrer JD, Mead S, Revesz T (2015) Review: An update on clinical, genetic and pathological aspects of frontotemporal lobar degenerations. Neuropathol Appl Neurobiol 41:858-881

Lotharius J, Falsig J, van Beek J, Payne S, Dringen R, Brundin P, Leist M (2005) Progressive degeneration of human mesencephalic neuronderived cells triggered by dopamine-dependent oxidative stress is dependent on the mixed-lineage kinase pathway. J Neurosci 25: 6329-6342

Mallapragada SK et al. (2015) Enabling nanomaterial, nanofabrication and cellular technologies for nanoneuromedicines. Nanomed: Nanotech Biol Med 11:715-729. doi:10.1016/j.nano.2014.12.013

Marker DF et al. (2013) The new small-molecule mixed-lineage kinase 3 inhibitor URMC-099 is neuroprotective and anti-inflammatory in models of human immunodeficiency virus-associated neurocognitive disorders. J Neurosci 33:9998-10010

Maroney AC et al. (2001) Cep-1347 (KT7515), a semisynthetic inhibitor of the mixed lineage kinase family. J Biol Chem 276:25302-25308

Masliah E, Ge N, Mucke L (1996) Pathogenesis of HIV-1 associated neurodegeneration. Crit Rev Neurobiol 10:57-67

Masliah E et al. (2005) Effects of $\alpha$-synuclein immunization in a mouse model of Parkinson's disease. Neuron 46:857-868

Mathiasen JR et al. (2004) Inhibition of mixed lineage kinase 3 attenuates MPP + -induced neurotoxicity in SH-SY5Y cells. Brain Res 1003:86-97

McGeer PL, EG MG (2004) Inflammation and neurodegeneration in Parkinson's disease. Parkinsonism Relat Disord 10:S3-S7

McGeer PL, Itagaki S, Akiyama H, McGeer EG (1988) Rate of cell death in parkinsonism indicates active neuropathological process. Ann Neurol 24:574-576. doi:10.1002/ana.410240415

Meairs S, Alonso A (2007) Ultrasound, microbubbles and the bloodbrain barrier. Prog Biophys Mol Biol 93:354-362

Moreland LW et al. (1999) Etanercept therapy in rheumatoid arthritis: a randomized, controlled trial. Ann Intern Med 130:478-486

Morgan D et al. (2000) A $\beta$ peptide vaccination prevents memory loss in an animal model of Alzheimer's disease. Nature 408:982-985
Munoz JL, Bliss SA, Greco SJ, Ramkissoon SH, Ligon KL, Rameshwar P (2013) Delivery of Functional Anti-miR-9 by Mesenchymal Stem Cell-derived Exosomes to Glioblastoma Multiforme Cells Conferred Chemosensitivity. Mol Ther Nucleic Acids 2:e126. doi: 10.1038/mtna.2013.60

Nowacek A, Gendelman HE (2009) NanoART, neuroAIDS and CNS drug delivery. Nanomed: Nanotech Biol Med 4:557-574

Nowacek A, Kosloski LM, Gendelman HE (2009) Neurodegenerative disorders and nanoformulated drug development. Nanomed (London, England) 4:541-555. doi:10.2217/nnm.09.37

Ozawa H, Noma S, Yoshida Y, Sekine H, Hashimoto T (2000) Acute disseminated encephalomyelitis associated with poliomyelitis vaccine. Pediatr Neurol 23:177-179

Pardridge WM (2005) The blood-brain barrier: bottleneck in brain drug development. NeuroRx 2:3-14

Pascual A, Hidalgo-Figueroa M, Piruat JI, Pintado CO, Gomez-Diaz R, Lopez-Barneo J (2008) Absolute requirement of GDNF for adult catecholaminergic neuron survival Nat Neurosci 11:755-761 doi: $10.1038 / \mathrm{nn} .2136$

Pathan SA et al. (2009) CNS drug delivery systems: novel approaches. Recent Pat Drug Deliv Formul 3:71-89

Perry VH, Nicoll JA, Holmes C (2010) Microglia in neurodegenerative disease. Nat Rev Neurol 6:193-201

Persidsky Y, Gendelman HE (2003) Mononuclear phagocyte immunity and the neuropathogenesis of HIV-1 infection. J Leukoc Biol 74: 691-701

Polymeropoulos MH et al. (1997) ) Mutation in the $\alpha$-synuclein gene identified in families with Parkinson's disease. Science 276:20452047

Popovich PG, Longbrake EE (2008) Can the immune system be harnessed to repair the CNS? Nat Rev Neurosci 9:481-493. doi: $10.1038 / \mathrm{nrn} 2398$

Ramaswamy S, Soderstrom KE, Kordower JH (2009) Trophic factors therapy in Parkinson's disease. Prog Brain Res 175:201-216. doi: 10.1016/s0079-6123(09)17514-3

Ransohoff RM, Brown MA (2012) Innate immunity in the central nervous system. J Clin Invest 122:1164-1171

Rivest S (2009) Regulation of innate immune responses in the brain. Nat Rev Immunol 9:429-439. doi:10.1038/nri2565

Salminen A, Ojala J, Kauppinen A, Kaarniranta K, Suuronen T (2009) Inflammation in Alzheimer's disease: amyloid- $\beta$ oligomers trigger innate immunity defence via pattern recognition receptors. Prog Neurobiol 87:181-194

Saporito MS, Brown EM, Miller MS, Carswell S (1999) CEP-1347/KT7515 , an inhibitor of c-jun N-terminal kinase activation, attenuates the 1-methyl-4-phenyl tetrahydropyridine-mediated loss of nigrostriatal dopaminergic neurons in vivo. J Pharmacol Exp Ther 288:421-427

Saporito MS, Hudkins RL, Maroney AC (2002) 2 discovery of Cep$1347 / \mathrm{Kt}-7515$, an inhibitor of the Jnk/Sapk pathway for the treatment of neurodegenerative diseases. Prog Med Chem 40:23-62

Schifitto G et al. (2007) Memantine and HIV-associated cognitive impairment: a neuropsychological and proton magnetic resonance spectroscopy study. AIDS 21:1877-1886

Schneeberger A, Tierney L, Mandler M (2015) Active immunization therapies for Parkinson's disease and multiple system atrophy Mov Disord 10.1002/mds.26377

Shi S, Wang Z, Qiao Z (2013) The multifunctional anti-inflammatory drugs used in the therapy of Alzheimer's disease. Curr Med Chem 20:2583-2588

Shih AY et al. (2006) Policing the police: astrocytes modulate microglial activation. J Neurosci 26:3887-3888. doi:10.1523/jneurosci.093606.2006

Skaper SD, Facci L, Giusti P (2014) Mast cells, glia and neuroinflammation: partners in crime? Immunology 141:314-327. doi:10.1111/ imm. 12170 
Sørensen LN, Reinert LS, Malmgaard L, Bartholdy C, Thomsen AR, Paludan SR (2008) TLR2 and TLR9 synergistically control herpes simplex virus infection in the brain the. J Immunol 181:8604-8612

Spillantini MG, Schmidt ML, Lee VM-Y, Trojanowski JQ, Jakes R, Goedert M (1997) $\alpha$-synuclein in Lewy bodies. Nature 388:839-840

Streit WJ, Graeber MB, Kreutzberg GW (1988) Functional plasticity of microglia: a review. Glia 1:301-307

Sui $\mathrm{Z}$ et al. (2006) Inhibition of mixed lineage kinase 3 prevents HIV-1 Tat-mediated neurotoxicity and monocyte activation. J Immunol 177:702-711

Thompson KA, Cherry CL, Bell JE, McLean CA (2011) Brain cell reservoirs of latent virus in presymptomatic HIV-infected individuals. Am J Pathol 179:1623-1629

Tobinick E, Gross H, Weinberger A, Cohen H (2006) TNF-alpha modulation for treatment of Alzheimer's disease: a 6-month pilot study. Med Gen Med: Medscape Gen Med 8:25

Verderio C, Matteoli, M (2001) ATP mediates calcium signaling between astrocytes and microglial cells: modulation by IFN-gamma. J Immunol (Baltimore, MD: 1950) 166:6383-6391

Whitley RJ (1990) Viral encephalitis. N Engl J Med 323:242-250

Williams DW, Eugenin EA, Calderon TM, Berman JW (2012) Monocyte maturation, HIV susceptibility, and transmigration across the blood brain barrier are critical in HIV neuropathogenesis. J Leukoc Biol 91:401-415

Wilson EH, Weninger W, Hunter CA (2010) Trafficking of immune cells in the central nervous system. J Clin Invest 120:1368-1379. doi:10. $1172 /$ jci41911
Wolak DJ, Thorne RG (2013) Diffusion of macromolecules in the brain: implications for drug delivery. Mol Pharm 10:1492-1504

Woodward E, Troedsson M (2015) Inflammatory mechanisms of endometritis. Equine Vet J 47:384-389

Young SK, Arndt PG (2009) c-Jun NH2-terminal kinase regulates lipopolysaccharide-induced pulmonary mononuclear cell recruitment via CCL2. Exp Lung Res 35:682-700

Zappia E et al. (2005) Mesenchymal stem cells ameliorate experimental autoimmune encephalomyelitis inducing T-cell anergy. Blood 106: 1755-1761. doi:10.1182/blood-2005-04-1496

Zhang G et al. (2016) The mixed lineage kinase-3 inhibitor URMC-099 improves therapeutic outcomes for long-acting antiretroviral therapy nanomedicine: nanotechnology. Biomark Med 12:109-122

Zhao Y et al. (2011) Active targeted macrophage-mediated delivery of catalase to affected brain regions in models of Parkinson's disease. J Nanosci Nanotechnol S4:pii-003

Zhao Y, Haney MJ, Gupta R, Bohnsack JP, He Z, Kabanov AV, Batrakova EV (2014) GDNF-transfected macrophages produce potent neuroprotective effects in Parkinson's disease mouse model. PLoS One 9:e106867. doi:10.1371/journal.pone.0106867

Zheng JC et al. (2008) HIV-1-infected and/or immune-activated macrophages regulate astrocyte CXCL8 production through IL-1 $\beta$ and TNF- $\alpha$ : Involvement of mitogen-activated protein kinases and protein kinase R. J Neuroimmunol 200:100-110 